Trastuzumab) hatten schwere Nebenwirkungen, sie führten bei $15 \%$ (vs. $7 \%$ ) der Patientinnen zum Therapieabbruch. Die häufigsten unerwünschten Wirkungen vom Grad 3 oder mehr waren Neutropenie (56\% vs. $60 \%$ ), Leukopenie (19\% vs. $21 \%$ ) und Diarrhö (18\% vs. $0 \%$ ).

Fazit: Die Studie widerlegt damit die in den Tyrosinkinase-Hemmer gesetzten Hoffnungen. „Die Fortsetzung einer
Trastuzumabbehandlung über die Progression hinaus bringt bessere Ergebnisse als die Umstellung auf einen Inhibitor der ErbB-Familie“, schlussfolgern Harbeck et al. Das Resultat entspricht zwei Studien mit dem TKI Lapatinib von 2015. Weder in MA.31 noch in CEREBEL war durch Lapatinib ein prognostischer Vorteil erreicht worden. „Trastuzumab-basierte Therapien bleiben damit die Therapie der Wahl für Patientinnen mit metastasiertem HER2-positivem Brustkrebs, bei denen Trastuzumab versagt hat", so lautet das Fazit der Autoren. Dr. Beate Schumacher

Harbeck $\mathrm{N}$ et al. Afatinib plus vinorelbine versus trastuzumab plus vinorelbine in patients with HER2-overexpressing metastatic breast cancer who had progressed on one previous trastuzumab treatment (LUX-Breast 1): an open-label, randomised, phase 3 trial. Lancet Oncol. 2016; 17(3):357-66.

\section{Vitamin-D-Mangel als Risikofaktor für eine onkogene HPV-Infektion}

\section{Erniedrigte Serumspiegel von 25-Hydroxy-Vitamin D korrelieren mit einer verminderten Abwehr gegen eine Reihe von respiratorischen Pathogenen sowie einem erhöhten Risiko für schwerere Krankheitsverläufe. Erstmals wurde jetzt die Assoziation einer erniedrigten Serumkonzentration mit einem erhöhten Risiko für eine Infektion mit onkogenen humanen Papillomviren beschrieben.}

$\mathrm{D}$ ie Bedeutung von Vitamin D im Knochenstoffwechsel ist hinlänglich bekannt. Als Indikator für den Vitamin D-Haushalt gilt das 25-HydroxyVitamin D (25(OH)D) im Serum. Die Serumspiegel von 25(OH)D können in Abhängigkeit von Alter, Rasse, Diät sowie der Dauer und Intensität der Sonnenexposition variieren. Es konnte nachgewiesen werden, dass Vitamin D auch eine unterstützende Wirkung auf das Immunsystem hat. 25(OH)D ist unter anderem an der Regulation der Expression von antimikrobiellen Peptiden beteiligt, die zur nichtspezifischen Abwehr von Pathogenen beitragen. Ausreichend hohe $25(\mathrm{OH}) \mathrm{D}$-Spiegel korrelieren mit einem Schutz vor Infektionskrankheiten, während erniedrigte Konzentrationen mit einem erhöhten Risiko für schwere Erkrankungsverläufe in Verbindung gebracht werden, beispielsweise bei Atemwegsinfektionen, die durch Influenzaviren oder durch das Respiratorische Synzytial-Virus verursacht werden. Auch beim Risiko einer aktiven Tuberkulose sowie der Rekonvaleszenz scheint Vitamin D involviert zu sein.

In dieser aktuellen Studie postulieren die Autoren, dass eine ausreichend hohe 25(OH)D-Konzentration eine protektive Rolle bei einer zervikovaginalen Infektion mit humanen Papillomviren (HPV) zukommt. In der Untersuchung wurde die Assoziation zwischen $25(\mathrm{OH}) \mathrm{D}$-Serumspiegeln und der Prävalenz von onkogenen HPV-Infektionen analysiert. Als Datengrundlage diente der National Health and Nutrition Examination Survey. Bei ausgewählten Frauen im Alter von 20-59 Jahren erfolgte eine HPV-DNA-Untersuchung mittels selbstentnommener Zervikovaginalabstriche. Zudem wurden sie hinsichtlich ihres Sexualverhaltens befragt.

Nach Berücksichtigung von Alter, Rasse und Ehestatus zeigten die Auswertungen der Ergebnisse, dass die Wahrscheinlichkeit für eine Hochrisiko-HPV-Infektion stieg einhergehend mit einer Abnahme der 25(OH)D-Serumkonzentration in Intervallen von 10 $\mathrm{ng} / \mathrm{ml}$ (adjustierte Wahrscheinlichkeit, $\mathrm{aOR}=1,14 ; 95 \%$-Konfidenzintervall [KI]: 1,02-1,27). Gleichermaßen erhöhte sich die Wahrscheinlichkeit für eine Infektion mit den HPV-Vazinetypen 16 und 18 bei stark erniedrigten $25(\mathrm{OH}) \mathrm{D}$ Spiegeln von $<12 \mathrm{ng} / \mathrm{ml}(\mathrm{aOR}=2,9$; $95 \%$-KI: 1,32-6,38) sowie bei erniedrig- ten Serumspiegeln von 12-19 $\mathrm{ng} / \mathrm{ml}$ $(\mathrm{aOR}=2,19,95 \%-\mathrm{KI}: 1,08-4,45)$ und auch bei unzureichenden Serumspiegeln von $20-29 \mathrm{ng} / \mathrm{ml}(\mathrm{aOR}=2,19$, 95\%-KI: 1,22-3,93) im Vergleich zu Frauen mit ausreichenden Serumkonzentrationen, die mit $\geq 30 \mathrm{ng} / \mathrm{ml}$ definiert wurden.

Shim J et al. Association between serum 25-hydroxyvitamin D level and human papillomavirus cervicovaginal infection in women in the United States. J Infect Dis. published 22 March 2016, doi:10.1093/infdis/jiw065

\section{Kommentar}

In dieser Studie wurde erstmals gezeigt, dass ein ausgeprägter Vitamin-D-Mangel mit einem erhöhten Risiko für eine zervikovaginale Infektion mit onkogenen HPV assoziiert ist. Im Umkehrschluss könnte dies wiederum bedeuten, dass ein ausgewogener Vitamin-D-Spiegel vor einer HPV-Infektion schützen könnte. Es wird vermutet, dass Vitamin D epitheliale Oberflächen auf der Haut, im Respirationstrakt und im Urogenitaltrakt gegenüber der Invasion von Bakterien und Viren unspezifisch schützt. Dies wäre eine Erklärung für die in dieser Untersuchung vorgestellten Befunde. Über den optimalen 25(OH)DSpiegel gibt es allerdings noch unterschiedliche Auffassungen, entsprechende Empfehlungen reichen von $20-29 \mathrm{ng} / \mathrm{ml}$ bis zu $\geq 30 \mathrm{ng} / \mathrm{ml}$.

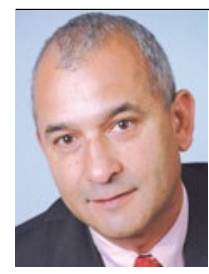

Prof. Tino F. Schwarz Zentrallabor, Stiftung Juliusspital Würzburg 